Templ at e-free synt hesi s and parti cle si ze cont rol of mesopor ous cal ci um car bonate

\begin{tabular}{|l|l|}
\hline 著者 & O SO Takahi ro, YANANAKA Shi nya \\
\hline $\begin{array}{l}\text { j our nal or } \\
\text { publ i cat } \mathrm{i} \text { on } \mathrm{t} \text { i t l e }\end{array}$ & Advanced Powder Technol ogy \\
\hline vol une & 29 \\
\hline number & 3 \\
\hline page $r$ ange & $606-610$ \\
\hline year & $2018-03$ \\
\hline URL & ht t p: //hdl . handl e. net /10258/00009945 \\
\hline
\end{tabular}




\title{
Template-free synthesis and particle size control of mesoporous calcium carbonate
}

\author{
Takahiro Oiso $^{\mathrm{a}}$, Shinya Yamanaka ${ }^{\mathrm{b}, *}$ \\ aDivision of Engineering, Muroran Institute of Technology, Mizumoto-cho 27-1, \\ Muroran 050-8585, Japan \\ ${ }^{b}$ College of Environmental Technology, Muroran Institute of Technology, \\ Mizumoto-cho 27-1, Muroran 050-8585, Japan \\ Corresponding author. Tel.: +81 14346 5747; fax: +81 143465701 \\ E-mail address: syama@mmm.muroran-it.ac.jp (S. Yamanaka)
}

\section{A B S T R A C T}

Controlling particle size is important in powder technology. Here we report a scalable production process of mesoporous calcium carbonate with a controllable particle size. We focus on the effect of the aggregation rate on the obtained particles. In this study, we change the particle concentration (1.2-12 mass\%) to control the aggregation rate and then obtained particles with relatively narrow range of particle size $(310 \pm 30-560 \pm 100$ $\mathrm{nm}$ ) and nearly identical specific surface areas and particle structures by template-free method. We proposed the aggregation model to describe the formation of meso-porous calcium carbonate.

Keywords: calcium carbonate, nanoparticles, aggregation rate, self-assembly. 


\section{Introduction}

The process of controlling particle properties (particle size, particle shape, etc.) is important in powder technology. Generally, powders have a particle size distribution with different shapes. Compared to coarser powders, handling of fine powders creates issues as the particle properties drastically change. For example, some problems such as increased frictional resistance, increased stickiness or adhesion, and scattering can arise. Shape variations affect the preparation time, quantitative capability, and product qualities. Hence, homogenization of particle size and the shape of raw material are necessary to improve product quality and handling properties.

Calcium carbonate is a common biomineral. The development of functional materials that mimic peculiar structures such as pearls and shells has attracted attention. Because biomineralization is a self-organization process in nature and can be effectively used for many materials, extensive research has occurred in the last few decade [1-3]. To design functional materials, it is necessary to understand the formation mechanism of calcium carbonate. The crystallization process is important in the synthesis and purification of calcium carbonate as well as in applications of solid materials. Research has been conducted to precisely control the crystal structure (calcite, aragonite, vaterite), particle size, particle shape, and pore structure by syntheses using interactions with macromolecules $[4,5]$ or additives [6-8]. Calcium carbonate that controls these particle characteristics is expected to be applied in various fields [9-11].

The most used industrial process of obtaining calcium carbonate involves the following steps [12]: (a) Calcination of limestone to produce quicklime and carbon dioxide; (b) A slaking process, where the quicklime is transformed into a slaked lime slurry (a $\mathrm{Ca}(\mathrm{OH})_{2}$ suspension), which is controlled by the addition of water; (c) A carbonation reaction, where $\mathrm{CO}_{2}$ is bubbled through an aqueous slurry of slaked lime. The carbonation reaction is the crucial step determining the particle characteristics of the obtained products. Especially, when calcium carbonate is applied as a filler, its particle size is important and has been controlled by adding additives [13-15]. Templates and additives are often used to control the particle characteristics (especially particle size and pore structure) of calcium carbonate and there is a problem that they must be removed.

In our research, we have improved the carbonation process, which is used in industry, and have advanced mass-production of mesoporous calcium carbonate by a template-free method in an organic solvent. Performing the carbonation reaction in an organic solvent yielded a calcium carbonate colloidal dispersion. Aging the obtained 
dispersion under various conditions gave the following results [16]: (1) When a colloidal solution with dispersed nanosized calcium carbonate is aged statically, the solution becomes cloudy after gelation. (2) The aging temperature greatly affects particle formation. For example, when the aging temperature is $20{ }^{\circ} \mathrm{C}$, egg-like shaped calcite particles (specific surface area: $\sim 200 \mathrm{~m}^{2} \mathrm{~g}^{-1}$ ) with particle sizes of $\sim 500 \mathrm{~nm}$ are obtained. When the aging temperature is $200{ }^{\circ} \mathrm{C}$, rod-like shaped vaterite particles (specific surface area: $\sim 65 \mathrm{~m}^{2} \mathrm{~g}^{-1}$ ) with a major diameter of $1.2-1.5 \mu \mathrm{m}$ are obtained. (3) In the aging process, the phase transition of the crystal and the aggregation of the colloid occur simultaneously. Consequently, the resulting particles have various shapes and crystal structures.

In this research, we focus on particle aggregation and attempt to synthesize mesoporous calcium carbonate with particle size controlled by template-free method. Concretely, we examine the influence of particle concentration, which is an aggregation rate-determining factor, based on the aggregation rate formula of the particle in solution [17]. As aggregation rate determining-factors, there are three parameters of temperature, viscosity, particle concentration. Controlling the temperature or the viscosity is difficult. It is especially challenging to realize a uniform system temperature during high-temperature aging. A mixture of crystal polymorphs and variations in shape are observed [16]. So, we conducted experiments focusing on particle concentrations, which are relatively easy to control among these determinants. Then we assess the particle properties of mesoporous calcium carbonate obtained from the calcium carbonate dispersion as a function of particle concentration.

\section{Experimental}

\subsection{Synthesis of mesoporous calcium carbonate in an organic solvent}

Calcium hydroxide (Nacalai Tesque), ethylene glycol (Kanto Chemical), and ethanol (Kanto Chemical) were used as received. Calcium carbonate was prepared by the carbonation of calcium hydroxide in an ethylene glycol-ethanol organic solvent mixture $[16,18,19]$. We placed 50.0-g calcium hydroxide in 450-g mixed solvent (with 30 mass \% ethylene glycol). $\mathrm{CO}_{2}$ was blended with $\mathrm{N}_{2}$ at a concentration of $30 \mathrm{vol} \%$, and the flow rate of gas was $1.0 \mathrm{~L} \mathrm{~min}{ }^{-1}$. The carbonation reaction temperature was controlled at $20{ }^{\circ} \mathrm{C}$ using a water bath. After 140 minutes of carbonation, the suspension was centrifuged for 20 minutes at a rate of $3540 \mathrm{G}$ to remove the unreacted calcium 
hydroxide.

The concentration of the calcium carbonate colloid dispersed in the supernatant liquid estimated from the thermal weight change was $\sim 12.0$ mass $\%$, the solid content separated and removed was 1.2 mass $\%$, and the yield of calcium carbonate was $89 \%$.

A predetermined amount of a solvent solution (ethanol:ethylene glycol ratio of 7:3) was added to the solution after completion of the reaction to adjust the particle concentration. When it was aged statically at $20{ }^{\circ} \mathrm{C}$, the colloidal solution became a cloudy sol through the gel. In this research, aging was completed when the solution became a cloudy sol. The cloudy suspension was washed twice with ethanol to eliminate the excess ethylene glycol. The suspension was then centrifuged at $3540 \mathrm{G}$ for 15 minutes. The supernatant was discarded, and the residue was dried in a vacuum for 12 hours. The resultant dry powder was used in the measurements.

\subsection{Evaluation of the products}

The specific surface area and the pore distribution of the product were measured at 80 points (adsorption side, 40 points; desorption side, 40 points) from a relative pressure between 0.025 and 0.9975 using a nitrogen adsorption instrument (manufactured by Quantachrome, AUTOSORB-1-c/MK2). SEM (JEOL, JSM-6380A) was used to observe the particle shape. X-ray diffraction (MultiFlex-120NP manufactured by Rigaku) was used to analyze the crystal structure. The ratio of vaterite was calculated from the peak intensity of the X-ray diffraction using Rao's equation [20].

$$
f_{v}=\frac{I_{110(\mathrm{~V})}+I_{112(\mathrm{~V})}+I_{114(\mathrm{~V})}}{I_{110(\mathrm{~V})}+I_{112(\mathrm{~V})}+I_{114(\mathrm{~V})}+I_{104(\mathrm{C})}}
$$

Here, $I_{104(C)}$ is the peak intensity of calcite. $I_{110(V)}, I_{112(V)}$, and $I_{114(V)}$ are the peak intensities of vaterite. To determine the particle size, we selected 100 particles arbitrarily from the SEM image and measured their Feret diameter.

\section{Result and discussion}

\subsection{Aggregation of calcium carbonate colloidal nanoparticles}

Our method yields a transparent calcium carbonate colloid solution. Generally, dispersion and aggregation of particles in the liquid phase are described by DLVO 
theory $[21,22]$. The total potential energy is expressed by the sum of the electrostatic interaction and the van der Waals interaction acting between the particles dispersed in the solution. The potential energy of the electric double layer is extremely small in a mixed solvent of ethanol and ethylene glycol due to the low dielectric constant of the solvent. Therefore, the force acting between the calcium carbonate colloidal nanoparticles in the liquid phase is primarily van der Waals interactions, and the colloidal nanoparticles spontaneously aggregate.

When two aggregated particles are considered as one and the aggregated particles are not redispersed (irreversible coagulation), the aggregation rate is expressed by equation (2) $[17]$

$$
-\frac{d N}{d t}=k^{\prime} N^{2}=\frac{4 k T}{3 \eta} N^{2}
$$

Here, $k^{\prime}$ is the aggregation rate constant, $N$ is the number of particles in a certain volume, $k$ is the Boltzmann constant, $\eta$ is the viscosity coefficient, and $T$ is the absolute temperature. From equation (2), temperature $T$, viscosity $\eta$, and particle concentration $N$ can be considered as factors determining the aggregation rate.

Figure 1 shows the SEM images of the calcium carbonate particles obtained at each particle concentration. At a low concentration, spherical particles with a particle size of $\sim 300 \mathrm{~nm}$ are observed. As the concentration increases, the particle size increases to $\sim 500 \mathrm{~nm}$.

Figure 2 shows the result of the XRD measurements. Calcite crystals are obtained for all conditions and a significant difference is not confirmed. Table 1 shows the evaluation results of the particle characteristics of calcium carbonate obtained under each condition. In all conditions, the obtained particles exceed the specific surface area value of calcium carbonate synthesized with the template $\left(\sim 50 \mathrm{~m}^{2} \mathrm{~g}^{-1}\right)$ [4], and have mesopores. Furthermore, the specific surface area is as high as $\sim 150 \mathrm{~m}^{2} \mathrm{~g}^{-1}$, excluding the highest concentration conditions.

Figure 3 depicts the relationship between the aggregation rate and the particle diameter. These results show that the larger the particle concentration, the larger the particle size. 

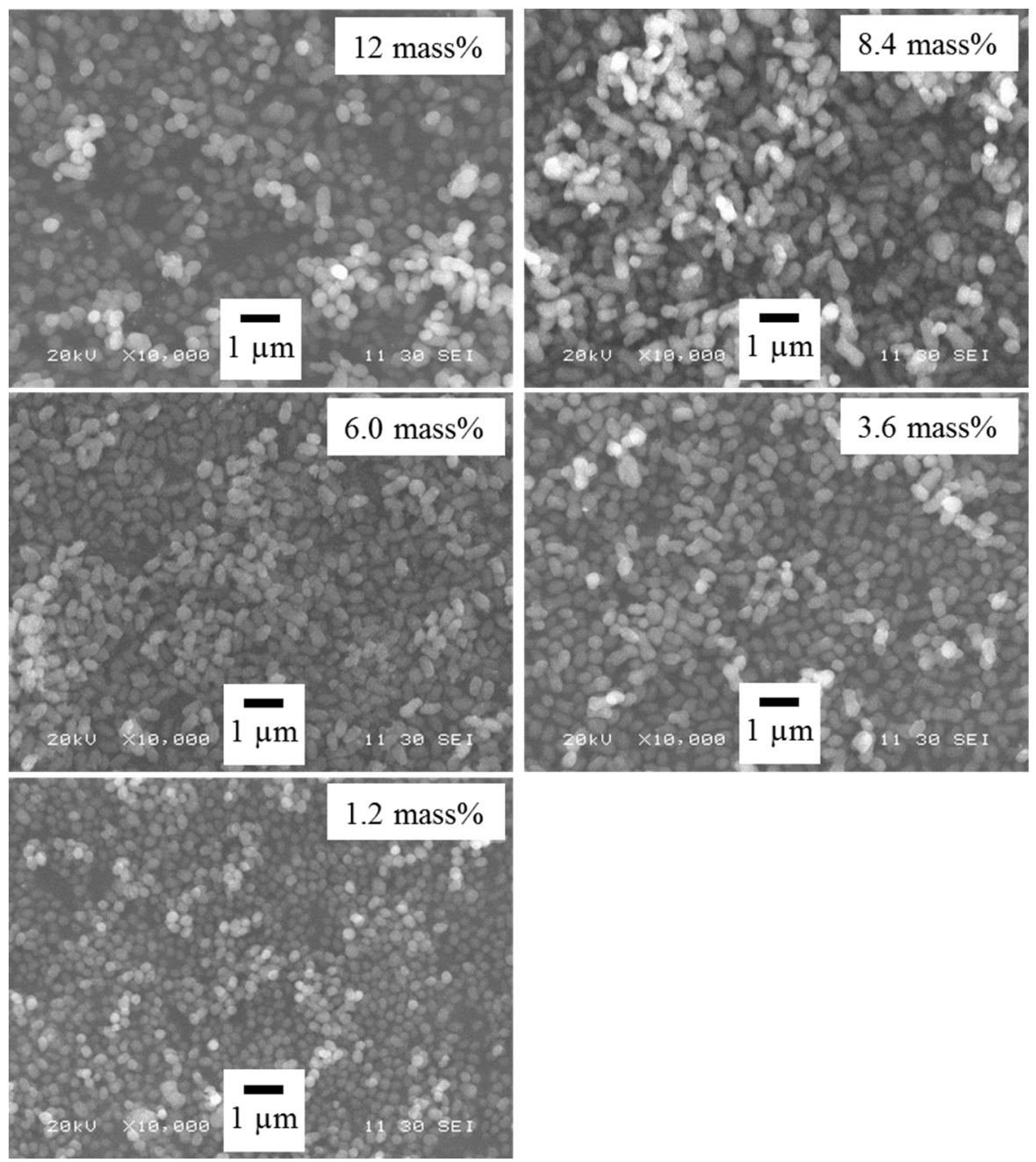

Fig. 1 SEM images of the particles obtained at each particle concentration 


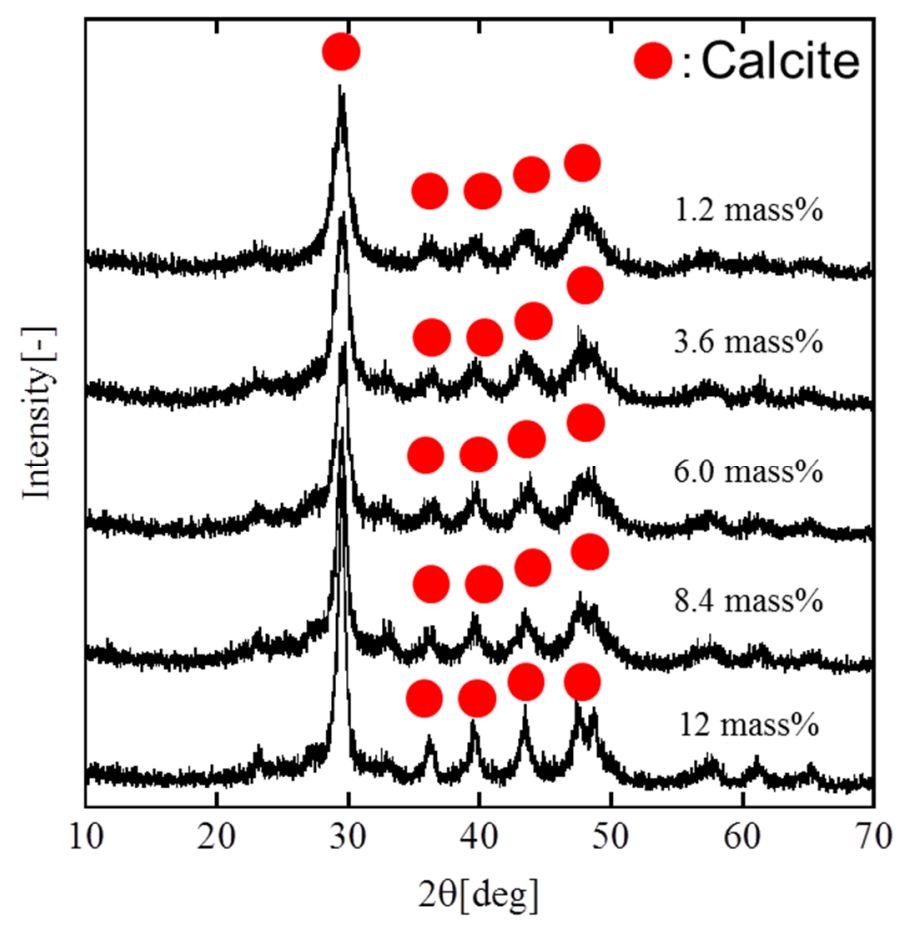

Fig. 2 XRD patterns of the particles obtained at each particle concentration

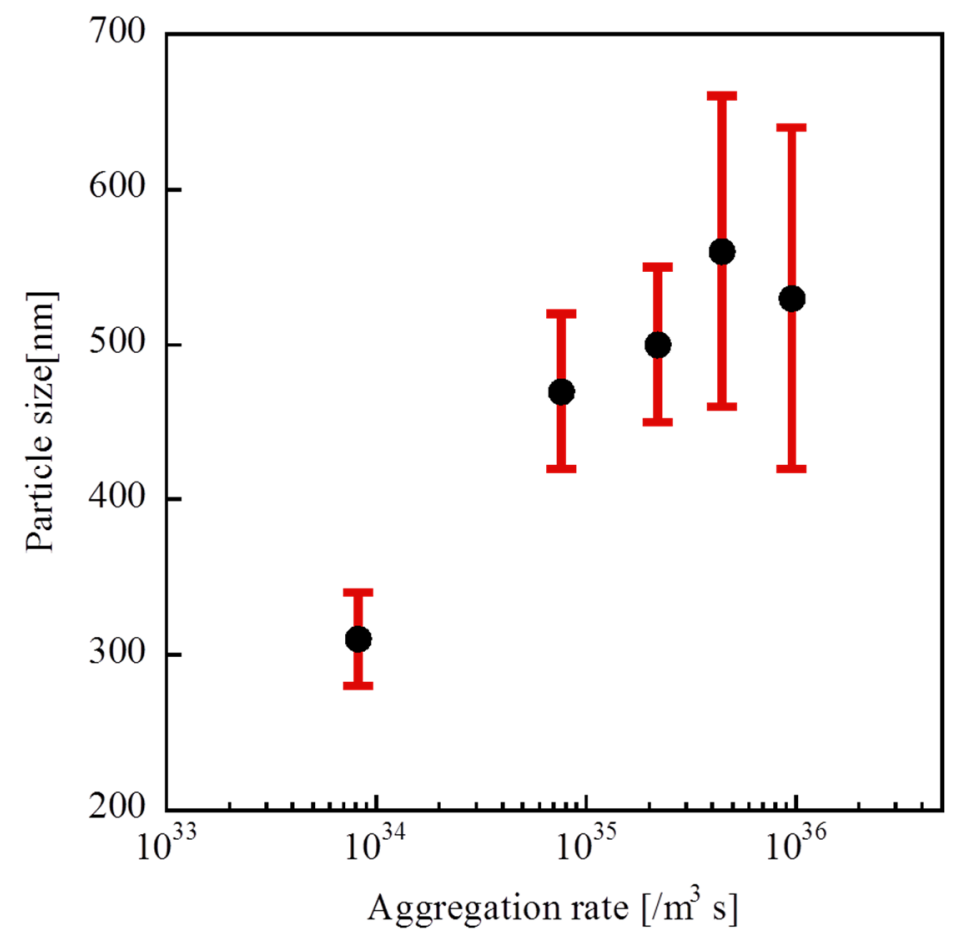

Fig. 3 Relationship between particle size and aggregation rate 


\section{Table 1}

Particle properties obtained at each particle concentration

\begin{tabular}{|c|c|c|c|c|c|c|c|c|}
\hline $\begin{array}{l}\text { Particle } \\
\text { concentration } \\
{[\text { mass } \%]}\end{array}$ & $\begin{array}{l}\text { Aging } \\
\text { time } \\
{[\text { day }]}\end{array}$ & $\begin{array}{l}\text { Amount of } \\
\text { particles } \\
{\left[\times 10^{25} / \mathrm{m}^{3}\right]}\end{array}$ & $\begin{array}{l}\text { Aggregation } \\
\text { rate } \\
{\left[\times 10^{33} / \mathrm{m}^{3} \mathrm{~s}\right]}\end{array}$ & $\begin{array}{l}\text { Particle } \\
\text { size } \\
{[\mathrm{nm}]}\end{array}$ & $\begin{array}{l}\text { Ratio of } \\
\text { vaterite } \\
{[-]}\end{array}$ & $\begin{array}{l}\text { Specific } \\
\text { surface } \\
\text { area } \\
{\left[\mathrm{m}^{2} / \mathrm{g}\right]}\end{array}$ & $\begin{array}{l}\text { Pore } \\
\text { volume } \\
{\left[\mathrm{cm}^{3} / \mathrm{g}\right]}\end{array}$ & $\begin{array}{l}\text { Crystallite size } \\
{[\mathrm{nm}]}\end{array}$ \\
\hline 1.2 & 10 & 6.29 & 8.22 & $310 \pm 30$ & 0.057 & 100.9 & 0.538 & 12.7 \\
\hline 3.6 & 6 & 19.2 & 76.4 & $470 \pm 50$ & 0.244 & 156.3 & 0.526 & 9.4 \\
\hline 6.0 & 3 & 32.5 & 220 & $500 \pm 50$ & 0.194 & 158.3 & 0.499 & 12.7 \\
\hline 8.4 & 2 & 46.3 & 446 & $560 \pm 100$ & 0.162 & 157.0 & 0.523 & 7.5 \\
\hline 12 & 1 & 68.0 & 959 & $530 \pm 100$ & 0.125 & 42.62 & 0.317 & 6.4 \\
\hline
\end{tabular}




\subsection{Influence of particle concentration on particle aggregation}

The calcium carbonate particles in solution after completion of the reaction spontaneously aggregate due to the van der Waals interaction. Therefore, we focused on the aggregation process and considered that changing the aggregation rate can control the obtained characteristics of particles.

During the aging stage, dissolution of the vaterite and subsequent reprecipitation form calcite. Thus, the water content in the colloidal dispersion should play an important role in the polymorphs. From this point of view, we found a fine balance between the aging time and water content; increasing the aging time or water content decreases the ratio of the vaterite phase [23]. In this study, a small amount of water was included in the colloidal solution due to the carbonation reaction $\left(\mathrm{Ca}(\mathrm{OH})_{2}+\mathrm{CO}_{2} \rightarrow\right.$ $\mathrm{CaCO}_{3}+\mathrm{H}_{2} \mathrm{O}$ ). Because a predetermined amount of a solvent solution was added to the colloidal solution (solid content was 12 mass $\%$, see section 2.1 ), the water content becomes low when the concentration of colloidal particles is low. However, a lower colloidal particle concentration requires a longer aging time. Consequently, the ratio of vaterite is maximized at a 3.6 mass \% particle concentration, as shown in Table 1.

By aging the solution after completion of the reaction at $20{ }^{\circ} \mathrm{C}$, the primary colloidal particles aggregate and form mesoporous calcium carbonate. However, the crystallite size on the (llll 04 ) plane of calcite crystals differs (Table 1). From this, it is inferred that particle growth and aggregation occur simultaneously in the low aggregation rate condition.

Regarding the crystallite size, the aging time plays a crucial role. Particle-growth mechanisms are generally divided into direct precipitation of simple solutes on the crystal face (Fig. 4, left) and aggregation mechanisms where primary particles form aggregates and then grow (Fig. 4, right) [24-26]. In this research, aggregation is the dominant reaction at high aggregation rates, but primary colloidal particle growth may also occur at low aggregation rates (Fig. 5). At a low aggregation rate (low concentration), we allotted sufficient time for primary particle growth before collecting the final aggregates. Consequently, it is inferred that the size of the produced particles and crystallite size differ.

Furthermore, the specific surface area and pore volume are small at the high aggregation rate condition. We previously demonstrated that calcium carbonate colloidal particles aggregate to form particles in our process [16, 23]. Based on previous results, the interstices of particles when forming particles become pores, providing 
mesoporous calcium carbonate. For a high particle aggregation rate, the particles collide, reducing or destroying the interstices.

Temporal changes are important in terms of morphology and particle size of the aggregates during the aging stage. Because visualizing the intermediate products in the aging process is extremely difficult in the native state, we are unable to show direct evidence regarding the particle size (and shape). Additionally, according to Meldrum and Cölfen [27], aggregation of nanocrystallites is very hard to control in terms of morphology and size of the final aggregate. Very recently, Nudelman et al. [26] provided the in vitro evidence for aggregation and transformation from amorphous calcium to a crystalline solid state using cryoTEM. We plan to investigate the relationship between particle size (and shape) and the aggregation rate. 
: Primary particle

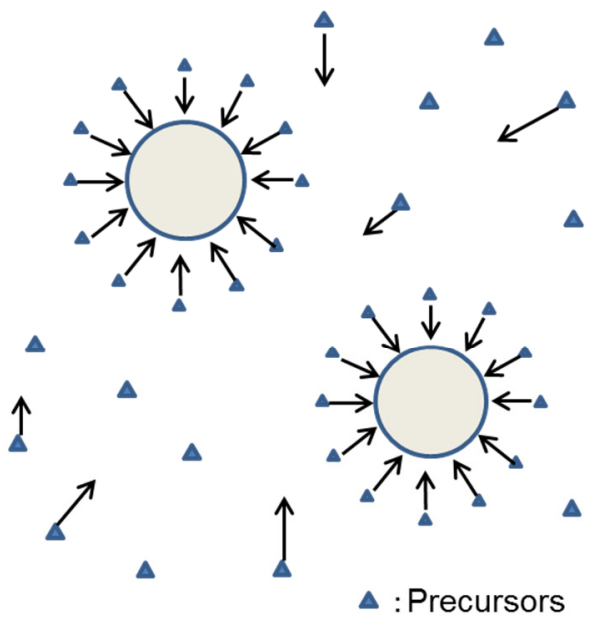

LaMer Mechanism

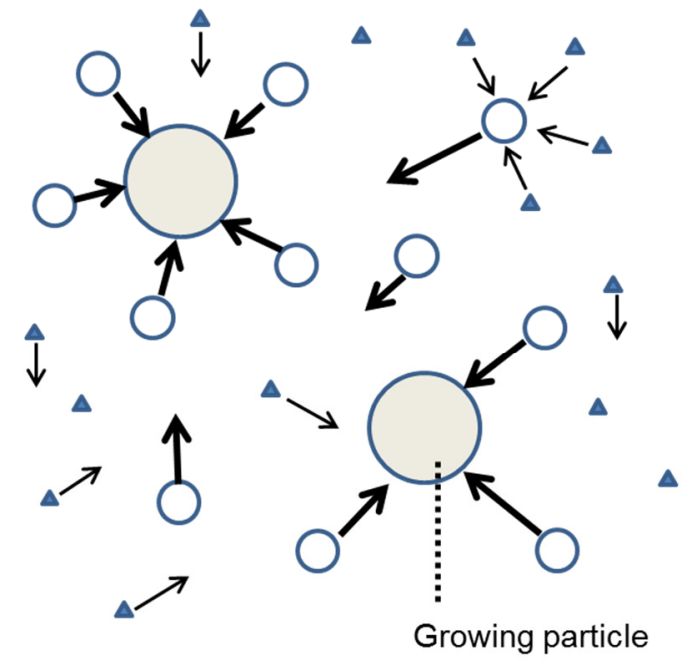

Aggregation Mechanism

Fig. 4 Particle growth mechanisms

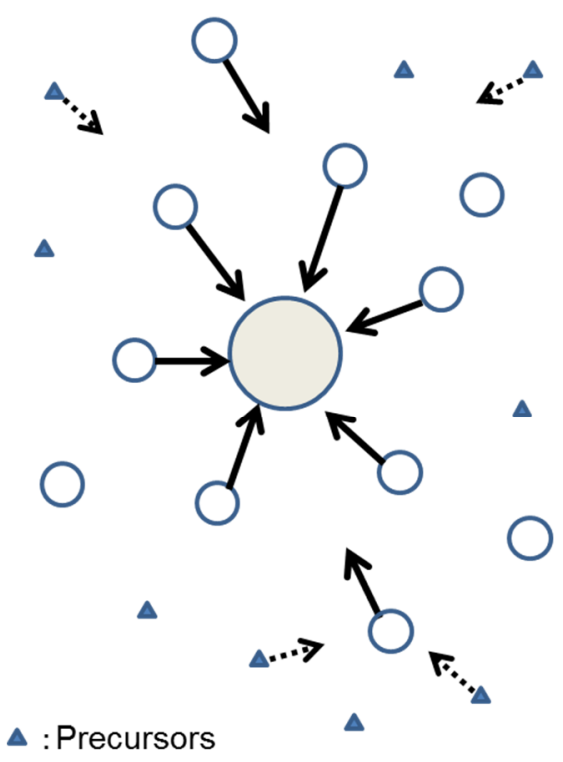

High aggregation rate

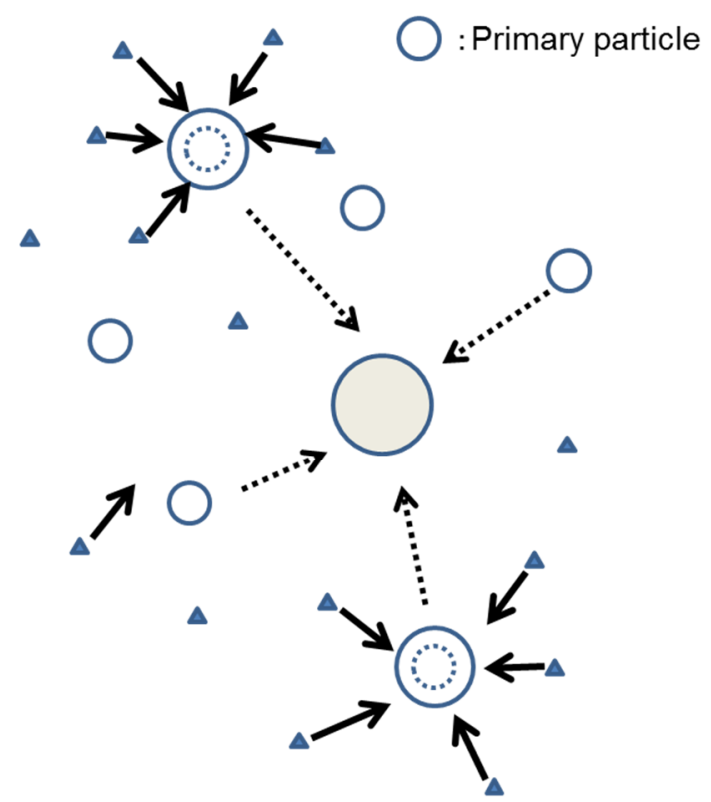

Low aggregation rate

Fig. 5 Particle-growth mechanisms by different aggregation rates 


\section{Conclusion}

We synthesized mesoporous calcium carbonate with a controlled particle size by the carbonation method in organic solvent. Calcite particles with particle size (300-500 nm) are obtained by adjusting the aggregation rate of the calcium carbonate colloid solution by changing particle concentration (1.2-12 mass $\%)$.

\section{Acknowledgment}

This work was supported by JSPS KAKENHI Grant Number JP16K13634. Authors thank Asahi Kohmatsu CO., LTD. for technical comments.

\section{References}

[1] W. Tongxin, H. Colfen and M. Antonietti, Nonclassical Crystallization: Mesocrystals and Morphology Change of $\mathrm{CaCO}_{3}$ Crystals in the Presence of a Polyelectrolyte Additive, J. Am. Chem. Soc., 127, (2005) 3246-3247

[2] A. Parayil , L. Wong, G. Subramanyam, R. Lakshminarayanan and S. Valiyaveettil, Synthesis and Characterization of Monodispersed Spheres of Amorphous Calcium Carbonate and Calcite Spherules, Cryst. Growth Des., 5, (2005) 1129-1134

[3] C. M. Fiona and S. T. Hyde, Morphological influence of magnesium and organic additives on the precipitation of calcite, J. Cryst. Growth, 231, (2001) 544-558

[4] G. Xiaohui, L. Liu, W. Wang, J. Zhang, Y. Wang and S. Yu, Controlled crystallization of hierarchical and porous calcium carbonate crystals using polupeptide type block copolymer as crystal growth modifier in a mixed solution, Cryst. Eng. Comm., 13, (2011) 2054-2061

[5] B. S. Gleb, D. V. Volodkin, A. M. Günther, A. I. Petrov, D. B. Shenoy, and H. Muhwald, Porous calcium carbonate microparticles as templates for encapsulation of bioactive compounds, J. Mater. Chem., 14, (2004) 2073-2081

[6] Y. Feng-Wen, C. Guo, X. Zhang and G. Yuan, Synthesis and characterization of spherical porous calcium carbonate with ordered secondary structures in the presence of polymer with double hydrophilic ionic moieties, Cryst. Eng. Comm., 14, (2012) 1554-1560

[7] K. K. Sand, J. D. Rodriguez-Blanco, E. Makovicky, L. G. Benning and S. L. S. Stipp, Crystallization of $\mathrm{CaCO} 3$ in Water-Alcohol Mixtures: Spherulitic Growth, Polymorph Stabilization, and Morphology Change, Cryst. Growth Des., 12, (2012) 
$842-853$

[8] Z. Shudong, M. Zhou, X. Lu, C. Wu, Y. Sun and Y. Xie, Macroscaled mesoporous calcium carbonate tetragonal prisms: top-down solid-phase fabrication and applications of phase-change material support martice, Cryst. Eng. Comm., 12, (2010) 3571-3578

[9] M. Yamamoto, H. Wakasa, H. Okada, Development of High Quality Porous Fillers (Part I ) -Study of the Effect of Particle Properties of Porous Fillers on Bulkiness and Opacity of Paper-, JAPAN TAPPI JOURNAL, 67(2), (2013) 185-191

[10]Z. Bartczak, A.S. Argona, R.E. Cohena, M. Weinberg, Toughness mechanism in semi-crystalline polymer blends: II. High-density polyethylene toughened with calcium carbonate filler particles, Polymer, 40, (1999) 2347-2365

[11]S. Sahebian, S.M. Zebarjad, J. Vahdati Khaki, S.A. Sajjadi, The effect of nano-sized calcium carbonate on thermodynamic parameters of HDPE, J. Mater. Process. Technol., 209, (2009) 1310-1317

[12]C. Domingo, J. G. Carmona, E. Loste, A. Fanovich, J. Fraile, J. G.-Morales, Control of calcium carbonate morphology by precipitation in compressed and supercritical carbon dioxide media, J. Crystal Growth, 271, (2004) 268-273

[13] Y. Sheng, B. Zhou, J. Zhao, N. Tao, K, Yu, Y. Tian, Z. Wang, Influence of octadecyl dihydrogen phosphate on the formation of active super-fine calcium carbonate, $J$. Colloid Interface Sci., 272, (2004) 326-329

[14]M. Vucak, M. N. Pons, J. Perid, H. Vivier, Effect of precipitation conditions on the morphology of calcium carbonate: quantification of crystal shapes using image analysis, Powder Technol., 97, (1998) 1-5

[15]D. S. Kim, C. K. Lee, Surface modification of precipitated calcium carbonate using aqueous fluosilicic acid, Appl. Surf. Sci., 202, (2002) 15-23

[16]S. Yamanaka, T. Oiso, Y. Kurahashi, H. Abe, K. Hara, T. Fujimoto and Y. Kuga, Scalable and template-free production of mesoporous calcium carbonate and its potential to formaldehyde adsorbent, J. Nanopart. Res., 16, (2014) 2266.1-2266.8

[17]W. I. Higuchi, R. Okada, G. A. Stelter, A. P. Lemberger, Kinetics of rapid aggregation in suspensions. Comparison of experiments with the Smoluchowski theory, J. Pham. Sci., 52, (1963) 49-54

[18]T. Yasue, A. Mamiya, T. Fukushima and Y. Arai, Synthesis and Characteristics of Amorphous Calcium Carbonate in Ethanol, Gypsum \& Lime, 198, (1985) 245-252

[19]T. Tatsuya, M. Fuji, M. Takahashi, C. Takai and M. Utsuno, Hollow Structure Formation Mechanism of Calcium Carbonate Particles Synthesized by the $\mathrm{CO} 2$ Bubbling Method, Cryst. Growth Des., 12, (2012) 771-776 
[20]M. S. Rao, Kinetics and mechanism of the transformation of vaterite to calcite, Bull. Chem. Soc. Jpn, 46, (1973) 1414-1417

[21]V. A. Parsegian, "Van der Waals Forces", Cambridge University Press, New York (2006)

[22]H. C. Hamaker, "The London-van der Waals Attraction between Spherical Particles", Physica, 4, (1937) 1058-1072

[23]S. Yamanaka, Y. Sugawara, T. Oiso, T. Fujimoto, Y. Ohira, Y. Kuga, Phase transformation of mesoporous calcium carbonate by mechanical stirring, CrystEngComm, 17, (2015), 1773-1777

[24]W. Stober, A. Fink, E. Bohn, Controlled growth of monodisperse silica spheres in the micron size range, J. Colloid Interface Sci., 26, (1968) 62-69

[25] V. K. LaMer, R. Dineger, Theory, Production and Mechanism of Formation of Monodispersed Hydrosols, J. Am. Chem. Soc., 72, (1950) 4847-4854

[26]J. M. Walker, B. Marzec, F. Nudelman, Solid-state transformation of amorphous calcium carbonate to aragonite captured by cryoTEM, Angew. Chem. Int. Ed., 56, (2017) 11740-11743

[27]F. C. Meldrum, H. Cölfen, Controlling mineral morphologies and structures in biological and synthetic systems, Chem. Rev., 108, (2008), 4332-4432 